\title{
Analysis on the Application Status and Existing Problems of Bekker 's Pressure-sinkage Model
}

\author{
Wenxuan Feng ${ }^{a, *}$, Jisheng Ma ${ }^{b}$ \\ Collage of Mechanical Engineering, Shijazhuang 050000, China \\ a1441790432@qq.com, b2972087569@qq.com
}

Keywords: Bekker theory, pressure-sinkage characteristics, track, tracked vehicle.

\begin{abstract}
The Bekker soil pressure-sinkage model is a classical theory to investigate the relationship between soil pressure and sinkage during vehicle travel. On the basis of the Bekker model, the descendants carried out a large number of experimental research, which played a significant role in promoting the development of vehicle-ground mechanics. In this paper, the principle of Bekker model is introduced briefly, and the application status and existing problems of Bekker model is discussed, and points out that, it is still an important direction for research to optimize and improve the bekker model.
\end{abstract}

\section{Introduction}

Bekker's theory of the vehicle-ground system is the classical theory of the field of vehicle-ground mechanics. After several decades of development, it has been widely used in military and civilian applications such as vehicle driving performance optimization, tracked vehicle track design.bekker was inspired from the plate loading test of the load-bearing capacity of the civil engineering, and the model of pressure-sinkage between vehicle and ground was proposed. And the bekker apparatus was designed, shown in Fig. 1. The Bekker model is a mechanics analysis of the interaction between vehicle and soil. By determining and measuring the appropriate soil properties, the simplified equation is derived from the vehicle and soil parameters ${ }^{[1]}$.

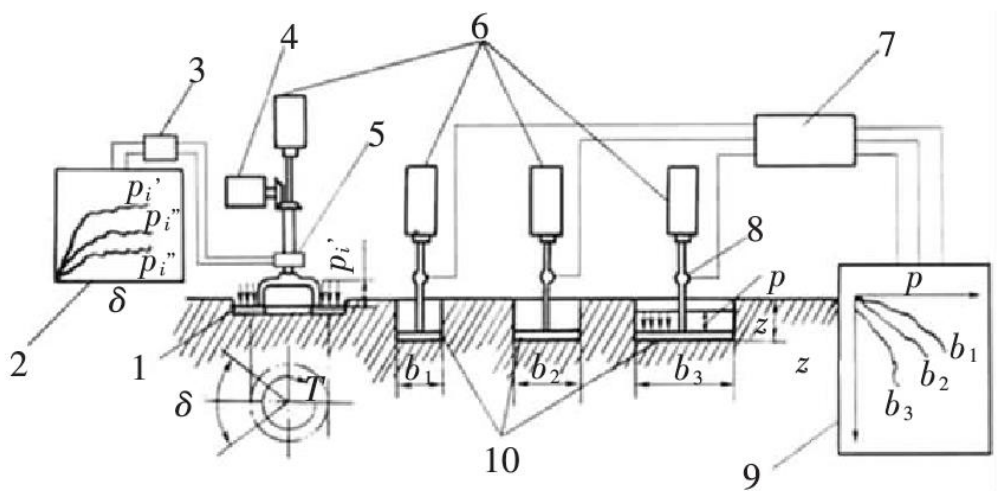

Fig.1 Bekker's apparatus

Fig.1 Schematic diagram of the Bekker's apparatus ${ }^{[2]}{ }^{21}$. Cutting ring 2,9. Recording tape 3,7. Amplifier 4. Torque motor 5. Torque and angle sensor 6. Loading cylinder 8. Pressure gauge 10. Through the flat plate

The Bekker model has undergone a large number of plate pressure-sinkage tests to summarize the Bekker formula:

$$
\mathrm{p}=\left(\frac{k_{c}}{b}+k_{\varphi}\right) \cdot z^{n}
$$

The parameters are: p-plate pressure; kc-soil cohesive deformation modulus; $\mathrm{k}_{\varphi}$ - soil friction angle deformation modulus; b-plate width; z-soil sinkage; n-soil deformation index. 
It can be seen from the Bekker formula that the relationship between the $\mathrm{p} \sim \mathrm{z}$ curves in the plate pressure-sinkage test is affected by four parameters. Where $K c, k_{\varphi}, n$ reflect the mechanical properties of the soil, and $b$ reflect the effect of the plate size on the p-z relationship.

In the field of vehicle-ground mechanics, the plate pressure-sinkage test is carried out according to the Bekker formula, and the relevant parameters of the soil are obtained, which is very important for the establishment of the vehicle-ground coupling system, especially the tracked vehicle-ground coupling system model Boundary conditions.

\section{Investigation status}

With the development of society and economic, production equipment is required to be mechanization in both military and civil field. Compared with wheeled vehicles, tracked vehicles has a better performance of cross-country. Under Consideration of the influence of track shoe, Li Jun, Zhou JingKai, Li Qiang ${ }^{[1]}$ conducted the investigation of performance of track sinkage, and the method of measuring the $\mathrm{p} \sim \mathrm{Z}$ curves under the conditions that $\mathrm{n}$ is non-integer was obtained. On the basis of the research of the paper ${ }^{[1]}$, Li Jun, Zhang $\mathrm{Yu}^{[2]}$ and others use the computer simulation technology to simulate the characteristics of the track shoe under certain soil conditions, so that the method of relying on theoretical analysis and experiment More diverse. Zeng Yihui, Zhou Yucai et al. ${ }^{[3]}$ used soil-water mixture instead of in-situ seabed sediment for pressure-sinkage test, combined with simulation technology, obtained the results of very close to the seabed sediment pressure-sinkage measurement data and used The intelligent algorithm improves the accuracy of the model and provides important boundary loading conditions for the simulation analysis of the tracked vehicle moving on the seabed.

\section{Some problems of Bekker pressure-sinkage model}

The soil has more complex mechanical properties and constitutive relationships than other materials. And the mechanical properties of the soil are influenced more obvious by the environmental conditions. During the soil mechanics experiment, it is relatively difficult to obtain the parameters of the in-situ soil, and the precision needs to be improved. There are some assumptions about the Bekker model. 1, The Bekker model reflects the soil pressure-sinkage characteristics during slow loading. 2, Bekker model assumes that the load distribution under the bottom of plate is uniform. 3, regardless the impact of the size and shape of the plate.

In the actual application process, the track vehicle driving process is a high-speed repeated loading process, the soil structure and state are different, and the bottom of the load plate load distribution is not ideal evenly distributed state. Therefore, there are deviation between theory of the Bekker model and actual situation in the using process. A lot of people or institutions engaging in relevant research do not fully consider this point.

\subsection{The differences between Bekker model loading rate and tracked vehicle driving process.}

The Bekker pressure-sinkage test is a quasi-static slow loading process, and the interaction between the track shoe and the ground is complicated when the tracked vehicle is running. However, when it is simplified as a pressure-sinkage model, the loading rate is much higher than that of the Bekker pressure-sinkage test. Therefore, the impact of the loading rate on the pressure-sinkage relationship should be studied when the Bekker pressure-sinkage model is used to study the pressure-sinkage characteristics of the interaction between the track shoe and ground. However, through the literature review, although the dynamic plate loading test has been studied and developed in civil engineering, but in the field of vehicle-ground mechanics, especially the tracked vehicle pressure-sinkage test research field, most still follow the Classical Theory of Bekker pressure-sinkage Model. The condition of Bekker pressure-sinkage model based on slow loading is not enough, which inevitably adversely affects the establishment of soil pressure-sinkage model. In the future research process, we should focus on the impact of loading rate on soil pressure-sinkage relationship, and improve the shell pressure-sinkage model, so that the study of pressure-sinkage characteristics between tracked vehicle and ground more closer to the actual situation. 


\subsection{Load distribution of plate bottom and shear performance having effect to the measurement of pressure-sinkage characteristics.}

The Bekker pressure-sinkage model assumes that the load on the bottom of the plate is evenly distributed, but whether the load is evenly distributed during the actual test, further research is needed. In addition, the soil shear performance will also affect the pressure-sinkage characteristics, the bekker model cannot reflect this. From the previous research results, the impact of soil shear stress on the pressure-sinkage is considered more, most researchers do not consider the load distribution in plate bottom. Wang Jiangying, Cao Wengui et al. ${ }^{[4]}$ considered the shear performance of the soil when analysis the interaction between deep-sea sediments and the track shoe, but it was difficult to measuring the pressure-sinkage characteristics and shear capacity at the same time in the actual experiment ${ }^{[4]}$. Although this paper improves the application of Bekker pressure-sinkage model in practice, considering the shear performance of soil, distribution under the bottom of load plate was not considered.

\subsection{Hysteresis of soil circulation loading.}

According to the theory of soil elastic-plasticity in higher soil mechanics, there is a hysteresis in the process of soil stress cycle ${ }^{[5]}$. In the case of stress, the strain includes two parts. One Part of the deformation is elastic, recorded as $\varepsilon_{1}$, the other part from the plastic deformation, recorded as $\varepsilon_{2}$. The total soil strain is:

$$
\varepsilon=\varepsilon_{1}+\varepsilon_{2} \text {. }
$$

Where the elastic strain is a recoverable part, the plastic strain is permanent and irrecoverable. During the cyclic loading process, the stress-strain curves of the soil loading process and the stress-strain curves at the time of unloading are not coincident. As shown in Fig. 2:

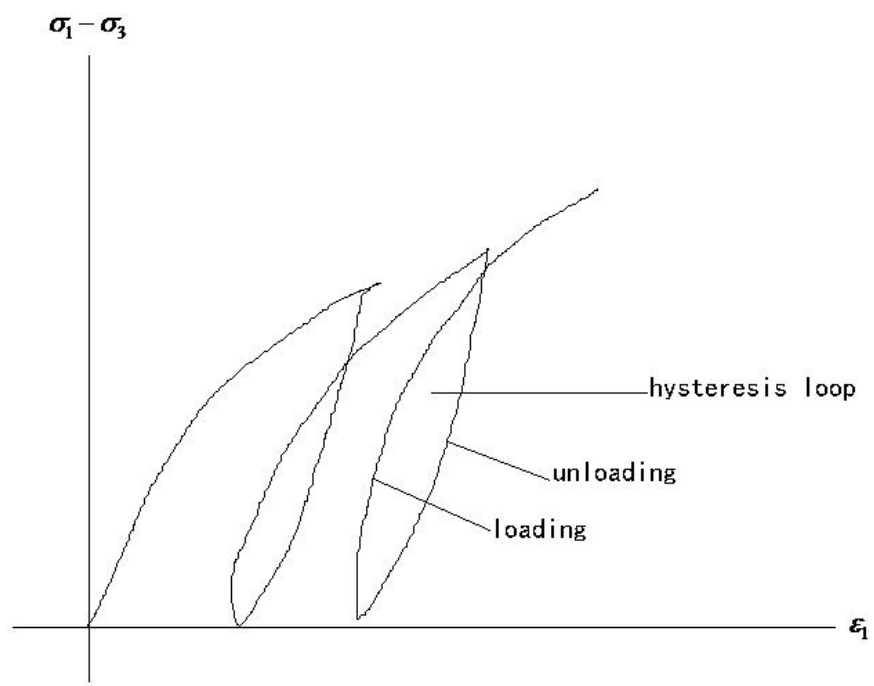

Fig. 2 Schematic diagram of hysteresis

During the loading process, the stress-strain curve is steep at the beginning, and gradually decreases with the increase of stress, which corresponds to the change of soil deformation modulus. During the unloading process, the stress level drops rapidly and then gradually slows down. With the loading cycle, the hysteresis loop repeats.

Tracked vehicles in the actual driving process, the interaction between track shoe and the ground can be considered as a cycle loading process. Therefore, in studying the pressure-sinking characteristics between tracked track and ground, we should consider the change of soil deformation modulus when soil stress is cycled. The traditional method of study only considers the pressure drop characteristics of the soil when loaded at one time. In the actual case, the soil deformation modulus of loading and unloading is not the same. 


\section{Conclusions and recommendations}

Bekker pressure-sinkage model is widely used in the study of soil pressure-sinkage characteristics between vehicle and ground. In particular, in the study of track performance and track structure optimization, based on the Bekker model and referenced to related parameter, the pressure-sinkage test provides important boundary load conditions for vehicle-ground system. In the application, though the Bekker model has been improved and optimized for specific problems, the problems about the loading rate, the load distribution of the plate and the hysteresis loops in the soil stress cycle are still worthy to be pay attention to.

\section{References}

[1]. Li Jun, Zhou Jingkai, Li Qiang.Study on track performance based on Bekker's theory [J]. Agricultural Equipment and Vehicles Engineering.Vol.(2012)No.2,p.14-16.

[2]. Li Jun, Zhang Yu, Zhou Jingkai,et al.Study on prediction method of trapping performance of large spiked track[J]. Agricultural Equipment and Vehicles Engineering. Vol. 51 (2013) No.4, p.33-36.

[3]. Zeng Yi-hong, Zhou Yu-cai, Liu Dao-cai,et al. A smart calibration model on track's pressure-sinkage characteristic of a tracked vehicle moving on soft seabed sediments [J].Journal of Central South University,Vol. (2013)No.20,p.911-917.

[4]. Wang Jiangying, Cao Wengui, Zhai Youcheng.Experimental study on the interaction between deep seawater and track [J]. Rock and Soil Mechanics. Vol.32 (2011) No.2, p.274-278.

[5]. Li Guangxin. Higher soil mechanics [M]. Tsinghua University Press, 2004, p. $42-43$. 